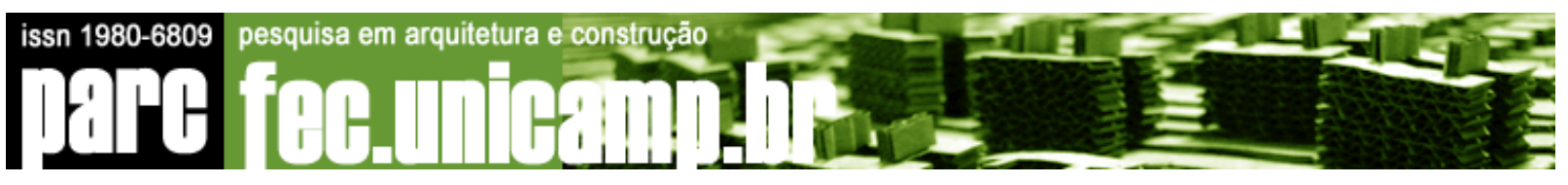

\title{
Design de componentes construtivos personalizados usando um método de desenho baseado nas restrições
}

Mass-customized constructive components design with the use of a constraint-based design method

\section{VELIZ, ALEJANDRO}

Arquitecto, Universidad Técnica Federico Santa María; Académico Escuela de Arquitectura, Facultad de Ingeniería Universidad de Magallanes, Punta Arenas, Chile.

aveliz@umag.cl

\section{GONZALEZ, LUIS FELIPE}

Arquitecto, Pontificia Universidad Católica de Chile; Académico Departamento de Arquitectura, Universidad Técnica Federico Santa María, Chile. Candidato a Doctor en Ciencias de la Ingeniería en la Bauhaus-Universität Weimar, Alemania.

\section{BARROS, LUIS PABLO}

Arquitecto, Universidad de Chile; Director Departamento de Arquitectura, Universidad Técnica Federico Santa María, Chile.

\section{Abstract}

This article describes a systematic implementation of an explored technology-transfer model, looking for an optimization of building, enlargement or equipments processes in the housing industry. For achieving that optimization, a heterogeneous group of manufacturing activities are studied according to the UN Classifications Registry (UN 2007), and their application in the chilean industry is evaluated. The explored model has been formulated according to Senn's (1992) method of prototype design, and the method of information systems development based on its life-cycle. Finally, by using a Visual Programming Language (VPL) interface, the model is applied for the formulation of the design problem of a customized constructive component. In this case, the component is a sanitary services pack. The proposal includes an experimental Graphic-User Interface (GUI), where each client configures the constructive components according to his particular requirements.

Keywords: Mass customization, Participative design, Constructive component manufacturing, Method of prototype design. 


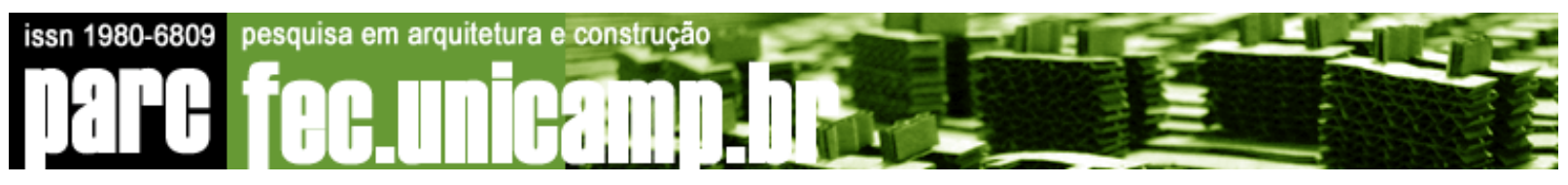

\section{Design de componentes construtivos personalizados usando um método de desenho baseado nas restrições}

\section{Introducción}

La automatización de procesos productivos bajo un modelo de personalización masiva se ha implementado con éxito en productos de pequeño formato. Sin embargo la baja innovación técnica en la construcción de viviendas impide una optimización de su proceso constructivo; generalmente el uso de tecnología se asocia a edificios únicos y de alto costo, así como el rol del arquitecto se asocia a obras particulares y exclusivas.

Tal como explica Sass (2005, traducción hecha por el autor), "urge un sistema automatizado para una rápida generación de alternativas de diseño y construcción (de viviendas). El sistema debe permitir una flexibilidad en el diseño y manufactura tal que las viviendas puedan variar en su función y forma a un mismo costo por superficie construida."

Dentro de las muchas alternativas para establecer un método formal de diseño (diseño basado en desempeño, diseño basado en componentes, diseño basado en casos, entre otros), el método de diseño basado en restricciones es especialmente útil al incorporarlo a medios de programación en un computador, al dividir un problema en partes manejables y acotadas, donde la determinación de las variables de diseño y de sus dominios de soluciones posibles finalmente permiten buscar dentro de sus combinaciones alternativas de diseño que respondan eficientemente a las restricciones iniciales. Este proceso de búsqueda puede ser iterado por múltiples usuarios y cuantas veces sea posible, a fin de buscar soluciones óptimas al problema.

El objetivo general de este trabajo fue la implementación de un modelo de producción masiva personalizada para componentes constructivos (fig. 01) manufacturados con rubros disponibles actualmente en el mercado chileno, apoyando procesos de planificación participativa para la ejecución, ampliación o remodelación de viviendas económicas, aumentando su calidad e involucrando al usuario final en la recopilación sistemática de requerimientos de diseño. 

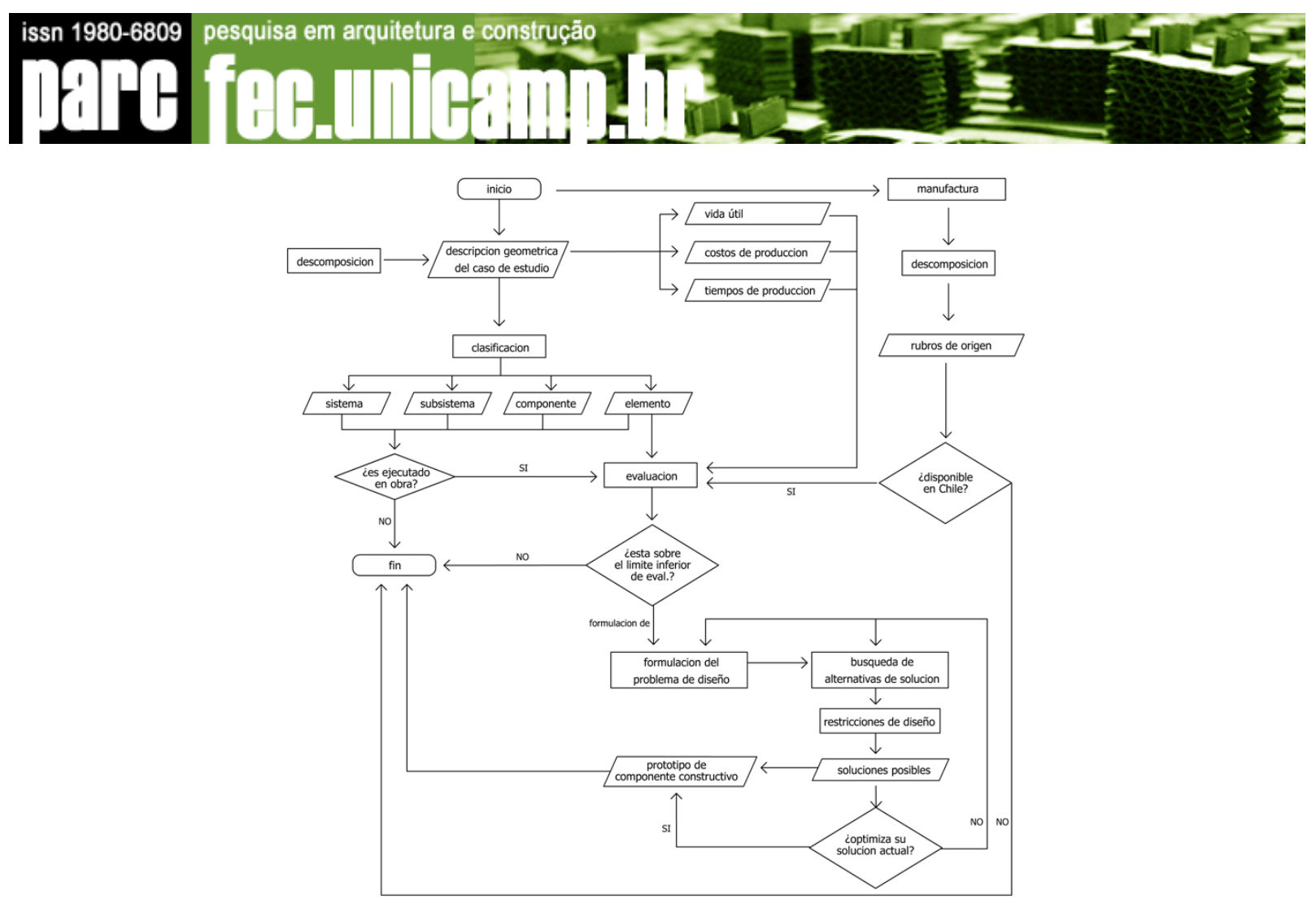

Fig. 01: Diagrama de flujo del sistema de información en el desarrollo de la investigacion.

La investigación realizada se puede dividir en 3 grandes etapas:

a. Búsqueda de condiciones iniciales del mercado chileno de construcción de viviendas y propuesta de optimización para su producción, basada en la fabricación de componentes constructivos personalizables con la ayuda de herramienas digitales y diseño participativo, asignándole a cada usuario una solución de calidad que responde a sus requerimientos (de manera semejante a lo propuesto por Duarte 2007) sin por ello incurrir en un aumento en los costos de construcción.

b. Formulación del problema de diseño de un componente particular -un muro de instalaciones sanitarias- y determinación de restricciones de diseño. Estas restricciones fueron programadas con una interfaz visual (VPL, Visual Programming Languaje) (fig. 02) y sus dominios de solución fueron acotados. Finalizada esta etapa, se realizó una búsqueda de alternativas de solución al problema de diseño. 


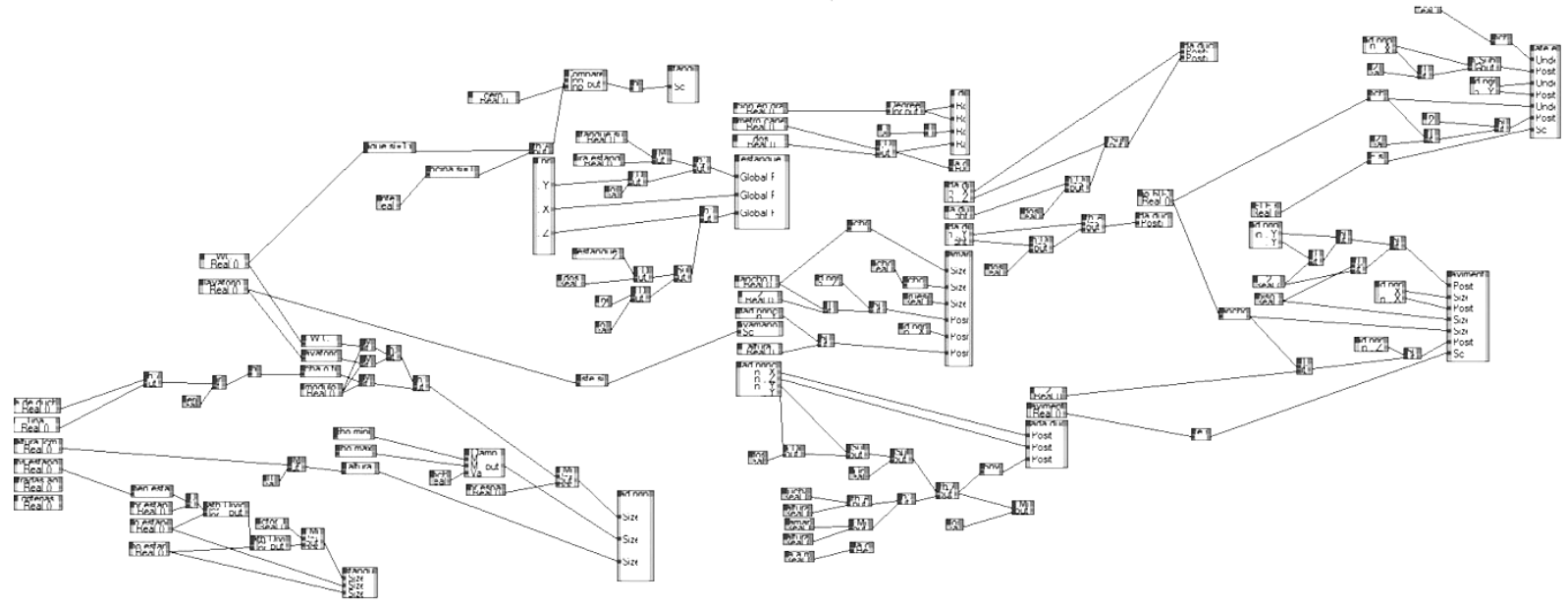

Fig. 02: Estructura de datos del problema de diseño. Elaboración propia.

c. Finalmente se proponen dos alternativas de fabricación para el producto diseñado rotomoldeado y termoformado de productos en plástico, según la clasificación n ²52 de actividades manufactureras de las Naciones Unidad, considerando la disponibilidad de rubros existentes en la industria chilena (SOFOFA 2007). El resultado final es un conjunto de 24 alternativas de diseño para un componente particular. Además, la propuesta incluye una interfaz experimental de usuario, en donde se ingresan los requerimientos particulares para la búsqueda de alternativas.

Este artículo se concentrará en la segunda etapa de la investigación: la formulación del problema de diseño para un muro de instalaciones sanitarias prefabricado, su división en componentes y restricciones de diseño, y su programación con la correspondiente búsqueda de soluciones personalizadas.

\section{La vivienda como caso de estudio}

Una baja innovación técnica en la construcción de viviendas impide una optimización de su proceso constructivo. Generalmente el uso de tecnología se asocia a edificios únicos y de alto costo, así como el rol del arquitecto se asocia a obras particulares y exclusivas. Sin embargo, la vivienda unifamiliar es el producto arquitectónico por excelencia; su alta demanda y alcance socio-económico la hacen un caso de estudio apto para implementar procesos de planificación participativa junto al usuario final. Dado que cada usuario tiene requerimientos particulares, se crea un escenario óptimo para la personalización masiva de componentes. 


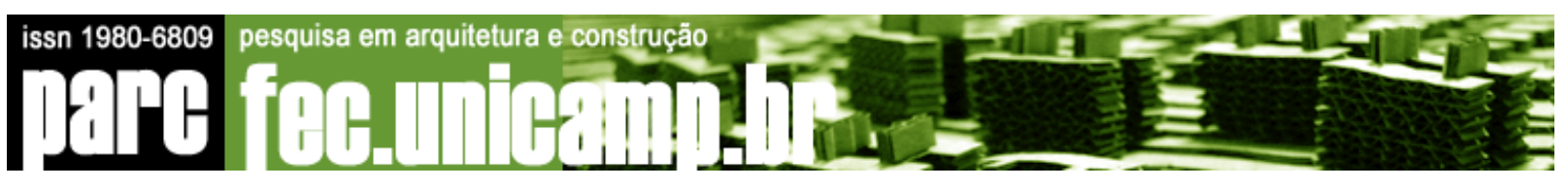

Una de las principales aproximaciones a un modelo productivo de personalización masiva está dada por el diseño de familias de componentes y productos. Este modelo se puede definir como un modelo rutinario, que compone el espacio de soluciones como respuestas a requerimientos explícitos (Gero 2007). A pesar de su alta demanda, la vivienda no se ha consolidado en Chile como un producto industrializado. Eso se debe a que la industrialización se asocia comúnmente a una pérdida de exclusividad del producto, el que tradicionalmente asigna una solución de diseño única para un conjunto variado de requerimientos.

\section{Formulación del problema de diseño del muro de instalaciones sanitarias}

Los sistemas de instalaciones sanitarias siempre han sido un tema de especial atención en el diseño de una vivienda. Su solución técnica define el uso y distribución de los recintos, ya que por ningún motivo es movible o reemplazable por otra solución. En la construcción de viviendas económicas los muros de instalaciones sanitarias suponen un problema constructivo por la alta cantidad de juntas y elementos que reúne. Ello provoca que las fallas en los muros y tabiques sean la primera causa de patologías en la construcción según estadísticas del Ministerio de Vivienda y Urbanismo (2005).

El muro a diseñar debe cumplir de manera efectiva su rol de separador de espacios y programas, de contener los ductos e instalaciones necesarias para el buen desempeño de los artefactos que un baño contiene y cuyos componentes lógicos (proceso de fabricación y distribución) utilicen el potencial productivo de un modelo de personalización masiva. Las siguientes condiciones cuantitativas luego serán formalizadas como restricciones de diseño: 
1. El muro de instalaciones tendrá dimensiones variables según un módulo dimensional de $70 \times 70 \times 60 \mathrm{~cm}$. , con un máximo de $210 \mathrm{~cm}$. en su ancho y $140 \mathrm{~cm}$ de pavimento.

2. El muro de instalaciones tendrá altura constante de $240 \mathrm{~cm}$.

3. Si el muro de instalaciones es adyacente a la cocina, entonces siempre se incorporará el estanque.

4. Si se incorpora el artefacto "WC", entonces siempre se incorporará el estanque.

5. Las salidas de ductos de agua potable serán a los $35 \mathrm{~cm}, 75 \mathrm{~cm}$ de altura con respecto al N.P.T.

6. Si se incorpora el artefacto "Lavatorio", entonces se incorporará una repisa.

7. Si se incorpora el artefacto "Pie de ducha", entonces siempre se incorporará el elemento "Pavimento".

8. Si se incorporan los artefactos "Pie de ducha" o "Tina", entonces siempre se incorporará una salida de agua potable adicional en los $180 \mathrm{cms}$ de altura.

9. Si se incorpora el elemento "Pavimento", entonces se incluirán las salidas de instalacion de alcantarillado correspondientes, las cuales podrán ser de 110 ó $15 \mathrm{~mm}$. de diámetro dependiendo del artefacto.

10. Se incorporará sólo 2 entradas de agua potable para todo el muro de instalaciones, 1 para el agua fría y 1 para agua caliente.

11. El volumen del estanque nunca podrá tener un espesor mayor al del muro, y su capacidad será de 6 lt.

12. Todas las entradas y salidas de ductos de agua potable o alcantarillado incorporarán griferías metálicas con hilo interior.

\section{Implementación: representaciones formales del problema}

A partir del diagnóstico de patologías de viviendas básicas (MINVU 2003) se propone el diseño de un muro de instalaciones sanitarias. Una vez establecido el problema de diseño, se definen los componentes del muro, sus atributos y las relaciones topológicas entre ellos. Este tipo de relaciones definen la relación física de adyacencia de cada parte con respecto a otra. Son 8 distintas, y se listan en el protocolo RCC8 Region Connection Calculus (Randell et al 2002). Este ejercicio permite determinar los requerimientos geométricos para cada una de las partes, lo que finalmente genera la estructura de datos cuantitativos del problema de diseño (fig. 3). 

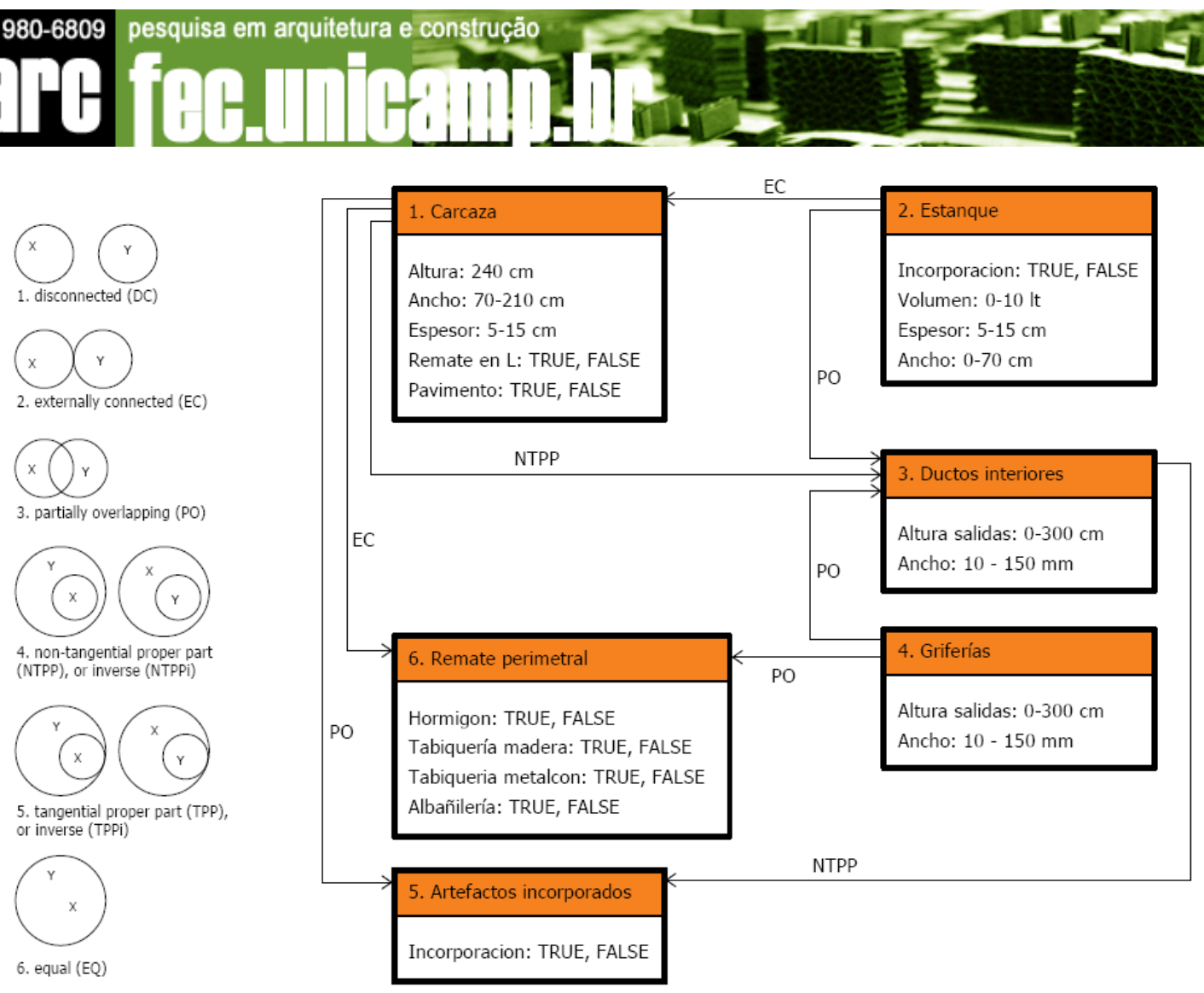

Fig. 3: Diagrama de relaciones topológicas y propiedades dimensionales para los componentes del muro de instalaciones sanitarias. Elaboración propia.

Con la información recopilada, se programó la estructura de datos del problema de diseño. Este procedimiento consiste en explicitar las relaciones geométricas entre los componentes, estableciendo relaciones de dependencia a partir de una serie de variables cuantitativas (fig. 4). La estructura del problema incluye implícitamente todas las soluciones posibles, siempre y cuando se haya establecido un correcto diagnóstico de éste y una serie coherente de relaciones entre las partes.

La estructura de datos se implementó en la interfaz de programación visual (VPL, Visual Programming Language) Xpresso® del software Cinema4D de MAXON®. La modelación del prototipo bajo este lenguaje incorpora implícitamente el espacio de soluciones posibles para el problema de diseño (Gonzalez 2007), a la vez que expresa en tiempo real las propiedades geométricas del modelo. 

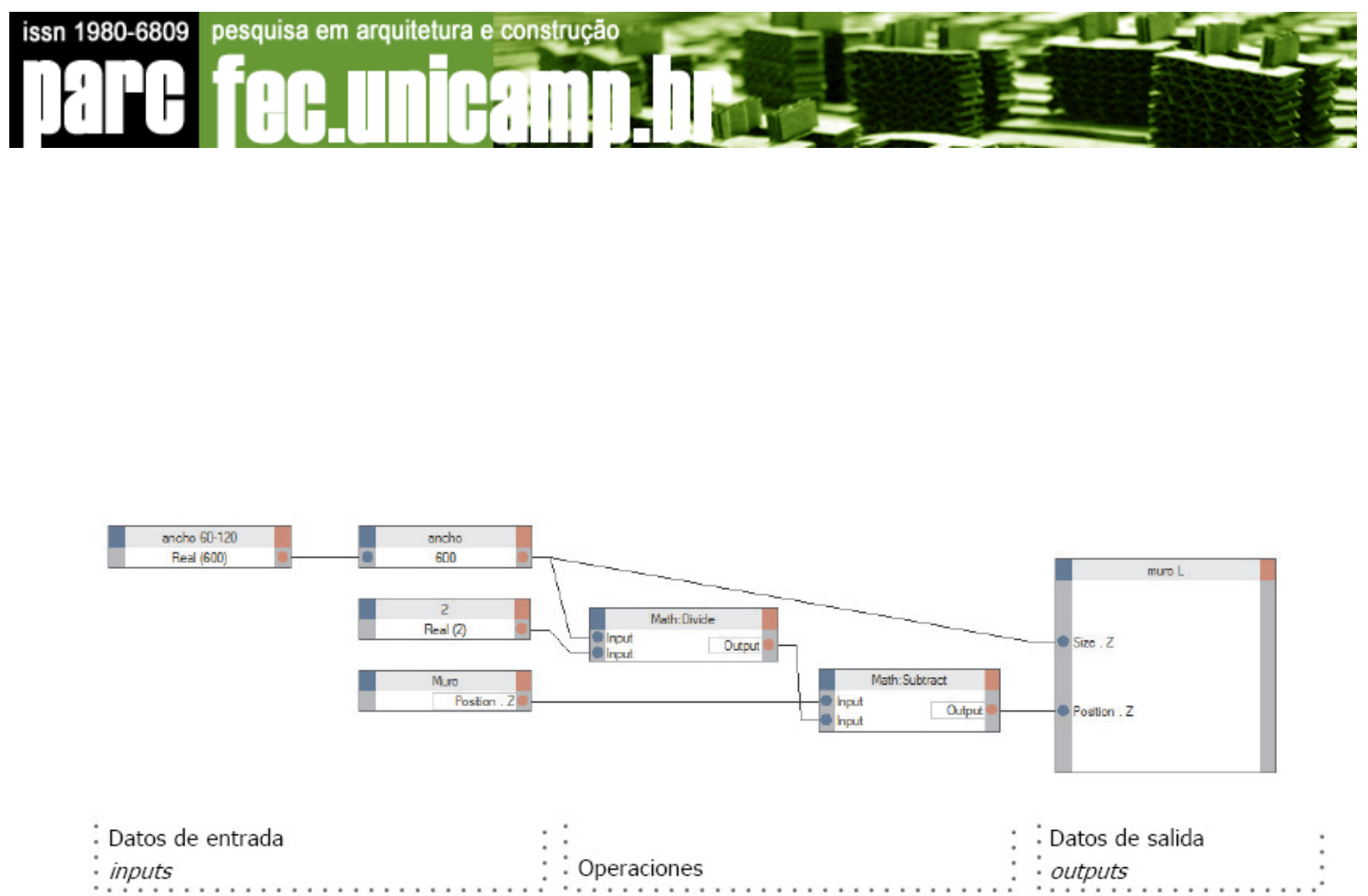

Fig. 4: Ejemplo de restricción de diseño para las propiedades "Size Z" y "Position Z" del componente "Muro L" del prototipo . Elaboración propia.

\section{Búsqueda de alternativas de solución al problema de diseño}

Mediante cambios iterativos sobre las variables del problema de diseño (sean estas dependientes o independientes) se buscaron alternativas de solucion al problema de diseño. Al no haber usuarios reales para este ejercicio, se utilizó un árbol de decisiones para diferenciar la inclusión, exclusión o variación en ciertos inputs de información, y así buscar la geometría del modelo resultante. En total, se exponen 24 alternativas (fig. 5). 

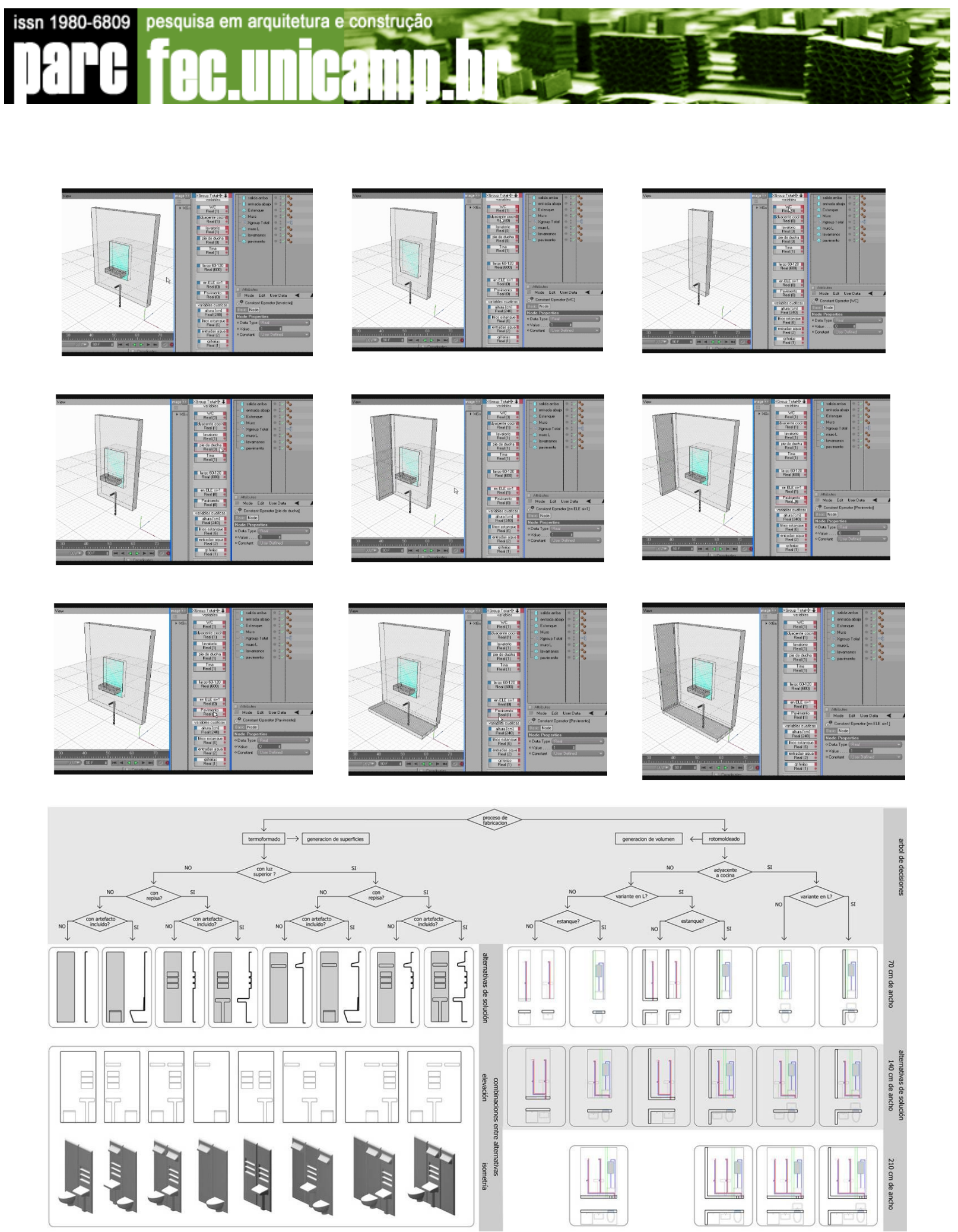

Fig. 5: Árbol de decisiones y alternativas de solución para el problema de diseño planteado. Cada alternativa responde a requerimientos particulares, manteniendo el proceso de manufactura.

\section{Discusión y conclusiones}




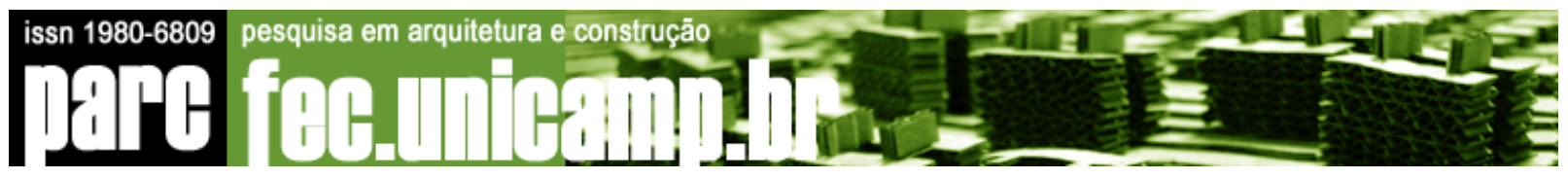

El desarrollo de la investigación implementa y finalmente valida una metodología propuesta para el desarrollo de componentes personalizados para viviendas en el mercado chileno. Este proceso de implementa gracias a una transferencia de tecnología desde el rubro de la manufactura de productos hacia el rubro de la construcción.

El principal impacto esperado es la diversificación de alternativas de componentes para la planificación participativa de viviendas, las que pueden ser aplicadas en la ejecución de viviendas sociales, viviendas nuevas o venta de departamentos "en verde" (venta previa al término de la obra, en la que el cliente puede realizar cambios al diseño original sin costos adicionales). Si bien aún no existe un restricción normativa que favorezca la planificación participativa en estos dos últimos casos, el aumento sostenido de la demanda lo convierte en un potencial campo de aplicación.

La investigación posiciona al arquitecto como un controlador del proyecto, no sólo al nivel de inspección técnica, sino que también con un conocimiento riguroso de los componentes lógicos: proceso de diseño, procesos productivos y procesos logísticos de transporte y montaje de elementos. Queda en evidencia la relación inequívoca entre los procesos de diseño y los procesos cognitivos de solución de problemas, enfrentando el problema de diseño con una estrategia de solución, dividiéndolo en partes y estableciendo las relaciones entre ellas para formular soluciones satisfactorias. Cabe mencionar que el método utilizado es una de muchas alternativas, como el diseño basado en componentes o el diseño basado en casos (estrategia ampliamente utilizada en ejercicios académicos de búsqueda de referentes y que ha sentado las bases para el desarrollo de tecnologías como la inteligencia artificial). Se presenta un enfoque sistémico y verificable para el proceso de diseño, generando información transmisible y discutible.

En ningún caso se pretende revalidar la visión renacentista del arquitecto-constructor, que se hace cargo de cada uno de los procesos que participan en el ciclo de vida de un proyecto. Actualmente la complejidad del proceso constructivo de cualquier obra es tal que se torna inabordable por una persona. A partir de ello han surgido tecnologías asociadas al manejo de información que optimizan dichos procesos. A pesar de lo aparentemente simple del proceso constructivo de una vivienda, se ha dejado en evidencia su potencial para el desarrollo y experimentación de innovaciones tecnológicas, a través del mejoramiento de los métodos de producción de sus partes individuales. 


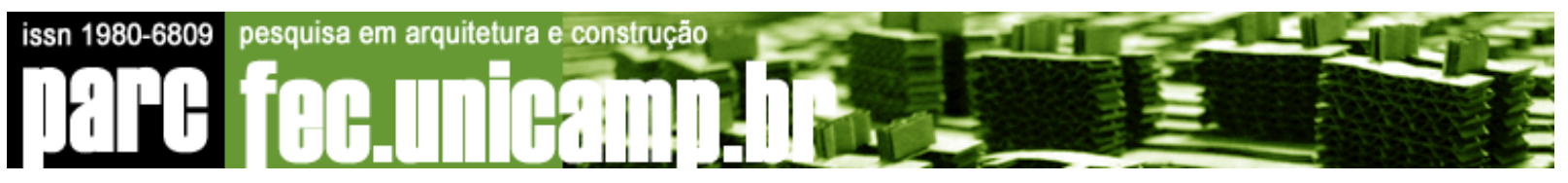

Finalmente, se valida el modelo explorado llegando a un conjunto de soluciones satisfactorias al problema impuesto, dejando en claro que ellas responden a los requerimientos de la estructura de datos modelada. Si bien es un modelo simple, la adición de componentes y relaciones hacen crecer exponencialmente la complejidad del modelo y por ende, las variables que intervienen en el proceso de búsqueda de soluciones.

Como valor agregado, se propone un uso masivo de la tecnología a través de componentes constructivos en pequeña escala para un mercado masivo, desmintiendo el prejuicio de un uso de la tecnología asociado a altos costos y en edificios exclusivos.

\section{Agradecimientos}

La presente investigación ha sido financiada por la Corporación de Desarrollo Tecnológico de la Cámara Chilena de la Construcción a través de su programa de Becas para Tesis de Pregrado, y patrocinada por los Departamentos de Obras Civiles y Arquitectura de la Universidad Técnica Federico Santa María. Se le agradece también al profesor Sr. Luis Felipe Gonzalez el apoyo continuo brindado durante el desarrollo y publicación de la presente investigación.

\section{Referencias}

Duarte, J. P. (2007) Personalizar a habitação em série: uma gramática discursiva para as casas da Malagueira do Siza. Lisboa, Fundação Calouste Gulbenkian e Fundação para a Ciência e a Tecnologia do Ministério de Ciência, Tecnologia e Ensino Superior de Portugal (FCT).

Colegio de Ingenieros de Chile AG (ed.) (2004), Plan estratégico País 2004: Sectores sociales, Seguridad social y Vivienda. Santiago, Chile.

Decreto Supremo $\mathrm{n}^{\circ 174}$ (2005, 30 de septiembre). Reglamento del Fondo Solidario de Vivienda. Santiago, Chile: Ministerio de Vivienda y Urbanismo:

Ekholm, A. (1994), A systemic approach to building modeling - Analysis of some objectoriented building product models. CIBW78 Workshop on integrated construction, Esbo, Finlandia. 


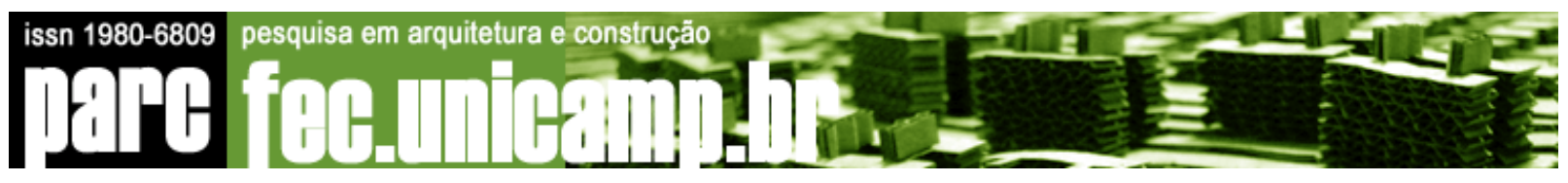

Federación Gremial de la Industria. Directorio Industrial. Consultado en octubre 2007 en http://www.sofofa.cl.

Gero, J., Sosa, R. (2007) Complexity measures as basis for mass customization novel designs. Key Centre of Design and Computing and Cognition, University of Sidney, Australia.

Gonzalez, L., Vargas, B. (2006) Foundations for a Constraint-Based Floor Plan Layout Support in Participatory Planning of Low-Income Housing. In Soza, P. ed. 10th SIGraDi Congress - Seminario Iberoamericano de Grafica Digital. Santiago de Chile, 283-287.

Ministerio de Vivienda y Urbanismo (2003) Diagnóstico de patologías en la edificación de viviendas básicas, Santiago, Chile: División Técnica de Estudio y Fomento Habitacional, Unidad de Tecnologías en la Construcción.

Ministerio de Vivienda Y Urbanismo. Estadísticas: Viviendas terminadas y subsidios pagados. Consultado en octubre 2007 en http://www.minvu.cl.

Randell, D. (2002) et al. A spatial logic based on regions and connections. 3rd International Conference on Knowledge Representation and Reasoning.

Sass, L. (2005). A production system for design and construction with digital fabrication.Disponible en http://ddf.mit.edu/projects/CABIN/cabin_mit_2005.pdf. Acceso en octubre/2008.

United Nations Statistics Division. Classifications Registry. Consultado en Agosto 2007 en http://unstats.un.org. 